\title{
Urinary Peritonitis due to Unrecognized Bladder Injury During Laparoscopy: A Case Report
}

\author{
Jacqueline Gascón ${ }^{\mathrm{a}}$, Maribel Acién ${ }^{\mathrm{a}}$, Pedro Acién ${ }^{\mathrm{a}, \mathrm{b}}$
}

\begin{abstract}
Bladder injury is a possible complication of laparoscopic surgery. If it is not identified, the subsequent clinical presentation is usually unclear and confusing. We report the case of a 31-year-old patient without an obstetric history. A laparoscopic ovarian cystectomy was performed and a bladder perforation due to the use of an accessory suprapubic trocar must have occurred. However, the perforation was not identified as such during the operation nor in the postoperative period. This injury subsequently caused unclear symptoms with diffuse and persistent abdominal pain, sometimes intense and focused on the right side, in the days following the surgery. The observation of the globe of the Foley catheter with trasvaginal ultrasound in an extravesical location suggested bladder injury with spilling of the urine and catheter into the peritoneal cavity. An immediate laparotomy was performed, revealing a small bladder perforation on the front pre-peritoneal face and another 1-cm perforation in the upper part of the bladder that were corrected. Postsurgical progress was then normal. A high urachal vesical extension or filled bladder, no visual control or a too-low introduction of the suprapubic trocar can produce an unrecognised bladder perforation. Attention must be paid to irritating and persistent abdominal pain of urinary peritonitis, presumed anuria corrected after the positioning of the Foley catheter, variable haematuria, leukocytosis, and eventual electrolytic alterations without fever. The key to the diagnosis of bladder injury is awareness of this clinical entity.
\end{abstract}

Keywords: Bladder injury; Suprapubic trocar; Surgical laparoscopy; Urinary peritonitis; Uroperitoneum

Manuscript accepted for publication February 8, 2013

${ }^{\text {aS }}$ ervice of Obstetrics and Gynaecology, University Hospital of San Juan; Department of Gynaecology, "Miguel Hernandez" University, Campus of San Juan, Alicante, Spain

${ }^{\mathrm{b}}$ Corresponding author: Pedro Acién, Departamento/Division de Ginecologia, Facultad de Medicina de la Universidad Miguel

Hernandez, Campus de San Juan, 03550 Alicante, Spain.

Email: acien@umh.es

doi: http://dx.doi.org/10.4021/wjnu83e

\section{Introduction}

Bladder injury is a possible complication of laparoscopic surgery that, if unidentified, may lead to a subsequently unclear and confusing clinical presentation. The patient may present with symptoms of intense and persistent abdominal pain due to urinary peritonitis as well as biochemical changes that introduce serious doubts and diagnostic difficulties while mimicking an acute renal failure [1-4]. We report a case of bladder injury due to perforation by an $11 \mathrm{~mm}$ accessory suprapubic trocar that was not diagnosed until four days after the laparoscopic surgery.

\section{Case Report}

A 31-year-old patient without an obstetric history was referred to our gynecology service for a $5.2 \mathrm{~cm}$ cyst on the right ovary and scheduled to undergo laparoscopic cystectomy. After preparation including vesical voiding, pneumoperitoneum and transumbilical laparoscopy, accessory trocars were introduced through the suprapubic area and left iliac fossae under laparoscopic vision. A cystectomy of the right ovarian cyst was performed without incident, and the cystic capsule was removed through an $11 \mathrm{~mm}$ suprapubic trocar-sheath and sent for pathological study. Normal genitals remained and the rest of the visualised abdominal cavity was normal. By the afternoon of the day of surgery, the gynaecologist was called due to persistent abdominal pain, vomiting and inability of the patient to urinate spontaneously. The patient was put on serum and a urethral Foley catheter was fitted letting the urine flow normally. The next day, there were only $225 \mathrm{~mL}$ of urine in the bag and haematuria was present. The patient was without fever but the continuous abdominal pain, focused at the hypogastry and right iliac fossae, remained. A transvaginal ultrasound was performed, revealing free fluid in the abdominal cavity. This fluid was more abundant toward the right iliac cavity and was interpreted as a remainder of the pelvic washing during the surgery. Blood analysis showed normal haemoglobin $(12.9 \mathrm{~g} / \mathrm{dL})$; however, there was leukocytosis $(18,200 / \mu \mathrm{L})$ 
with neutrophils at $91.4 \%$, and the urinary sediment demonstrated frank haematuria and leukocytes. The pain persisted and the Foley catheter was maintained together with the administration of analgesics.

On the following day, there was still continuous abdominal pain in spite of usual analgesics. A new abdominal examination revealed a soft abdomen, but there was slight pain in the hypogastric area, iliac fossae, and right flank with signs of peritoneal irritation. However, there was no fever or any other suggestive clinical data. The urine was now clear and blood analysis showed haemoglobin at $13 \mathrm{~g} / \mathrm{dL}$ and a leukocyte count of $18,800 / \mu \mathrm{L}$, with $87 \%$ neutrophils. The Foley catheter was removed, and some hours later, the patient spontaneously urinated a very small quantity but then called again due to intense pain. Thus, the Foley catheter was replaced and twelve hours later the diuresis reached 2,000 $\mathrm{mL}$. On the following day, the patient was better and showed good diuresis, but due to the persistent pain and occasional haematuria, a consultation with the urologists was requested. The blood analysis showed levels of haemoglobin at $11.7 \mathrm{~g} /$ $\mathrm{dL}$, leukocytes at $12,500 / \mu \mathrm{L}$, neutrophils at $79 \%$, creatinine at $1.1 \mathrm{mg} / \mathrm{dL}(\mathrm{N}: 0.6-1.3)$, sodium at $134 \mathrm{nmol} / \mathrm{L}(\mathrm{N}: 136-$ $145)$, calcium at $7.6 \mathrm{mg} / \mathrm{dL}(\mathrm{N}: 8.8-10.1)$, normal $\mathrm{Cl}$ and $\mathrm{K}$, and total proteins at $5.3 \mathrm{~g} / \mathrm{dL}(\mathrm{N}: 6.4-8.2)$. Urinary sediment showed increased red blood cells, leukocytes, and cylinders. The urologist who attended the patient ordered the removal of the Foley catheter since there were no evident abnormalities in the urinary tract. In the afternoon of the same day, the gynaecologist was required again due to the patient's intense and diffuse abdominal pain, vomiting, as well as her inability to urinate since the Foley catheter removal. She adopted an antialgic position and her abdomen was soft, with pain in response to deep palpation and signs of peritoneal irritation, but without fever. The Foley catheter was placed again, and very little urine was released. A transvaginal ultrasound was performed and free peritoneal fluid was observed but when the Foley catheter was moved, it seemed to be located in an extravesical location, suggestive of a bladder injury with spilling of the urine and catheter into the peritoneal cavity. An abdominal tomography was also performed and, again, a bladder break was suspected due to the presence of abundant free intraperitoneal fluid, bladder with scarce repletion, and the intraperitoneal location of the globe of the Foley catheter.

Surgical correction was then indicated but before, introduction of radiologic contrast was done, finding spilling of the contrast into the peritoneal cavity as well as preperitoneally. Therefore an immediate minilaparotomy was then performed, revealing a small bladder perforation on the front preperitoneal face and another $1 \mathrm{~cm}$ perforation in the upper part of the bladder. We thereby confirmed a double perforation of the bladder in its hypogastric urachal extension due to the passage of the accessory suprapubic trocar (entrance and exit). Besides, this trocar seemed to have been introduced too-low through hypogastric area during laparoscopy.
Both vesical tears were repaired and subsequently, the rest of the abdominal cavity was examined and washed. Then, a permanent Foley catheter was left in the bladder for four days. Post-surgical progress was normal and the patient was discharged from hospital 4 days after laparotomy. The subsequent control examinations were urologically and gynecologically normal.

\section{Discussion}

Laparoscopic surgery is associated with known complications, including bleeding or perforation of hollow organs such as the bowel, bladder, or ureters $[2,5]$. Approximately half of all complications occur due to insertion of trocars [6]. The bladder is particularly vulnerable to such an injury due to its location, distensibility, and thin wall $[2,3,7]$. The number of reported incidents of bladder injury varies. It is less than $0.2 \%$ in the case of diagnostic laparoscopy $[5,8]$, but more frequent in operative laparoscopy (up to $8 \%$ in laparoscopic-assisted vaginal hysterectomy) and frequently caused by sharp electrosurgical dissection [5, 8]. Jelovsek et al [9] concluded that the overall incidence of injury to the lower urinary tract in 126 consecutive total laparoscopic hysterectomies was $4.0 \%$, but Lafay Pillet et al [10] had a rate of bladder injuries of $1 \%$ in 1,501 procedures, decreasing with the surgeon experience and others report even less [11]. Although the majority of bladder injuries are detected at the time of surgery, either directly by the surgeon seeing urinary spilling or by the anaesthetist noticing gas or blood in the urine bag [7, 12], Jelovsek et al [9] recommended the performance of cystoscopy with intravenous indigo-carmine dye at the time of total laparoscopic hysterectomy. Naturally, it is a standard practice to empty the bladder prior to laparoscopy in order to improve the visibility of pelvic organs; but this is also important for limiting the risk of bladder injury $[2,3]$. If detected, the subsequent repair of bladder injuries observed during the operation can also be made via laparoscopy [13, 14].

Cases that go unnoticed during surgery can later present with serious complications that require laparotomy or other additional major surgery. This happened in two cases of bladder perforation that occurred in 953 consecutive cases of major operative laparoscopy reported by Saidi et al [12]. In cases of diagnostic laparoscopy or minor surgery without hysterectomy, bladder injuries are very unusual. They are sometimes caused by the insertion of the Veress-needle, but this injury does not require surgical repair. However, Darmon et al [15] reported a bladder injury during a laparoscopic procedure due to the presence of an urachal anomaly, with the bladder reaching the umbilicus. One of the accessory trocars perforated the bladder in this unusual position. A laparotomy was performed, revealing the urachal anomaly. The bladder injury was repaired, and the patient discharged 
without sequelae 12 days later. Our case seems similar to that one with regard to the mechanism of bladder injury.

The morbidity of unknown bladder injury is high and urinary peritonitis symptomatology is often non-specific [3, 16]. Due to their limited experience with similar injuries, physicians often have a low index of suspicion in such cases; thus, the diagnosis of the injury may be delayed by several days $[2,3,5,16]$. Patients may present with abdominal pain, low back pain, leukocytosis, and signs of peritoneal irritation or peritonitis 1 - 4 days after bladder injury [2]. In our case, as in others, low abdominal pain was present as early as 6 - 8 hours after surgery, improved with the placement of a Foley catheter, and worsened over subsequent days [8], especially when the Foley catheter was removed. If suspected, the diagnosis of bladder perforation can be confirmed radiologically by extravasation of contrast from the bladder [16]. Additionally, peritoneal fluid and even extravesical placement of the globe of the Foley catheter can also be seen under transvaginal or abdominal ultrasound.

On the other hand, profound disturbances in serum electrolytes and acid-base status (elevated serum urea, creatinine, and potassium, decreased serum sodium and $\mathrm{CO}_{2}$ content, and development of metabolic acidosis) are consistent findings among patients with intraperitoneal bladder rupture [1]. When urine enters the peritoneal cavity, reverse autodialysis occurs. Urea and creatinine diffuse down their concentration gradients into the blood, producing a characteristic biochemical profile of pseudo-renal failure. This appears within 24 hours of undiagnosed bladder rupture. The rapid early rise in creatinine suggests peritoneal urinary resorption rather than true acute renal failure. Some authors believe this to be diagnostic [17] but our patient did not present it. The low serum sodium associated with a biochemical profile otherwise consistent with dehydration and renal failure should suggest the diagnosis of urinary peritonitis [18]. Wystrychowski et al [19] also noted that the uroperitoneum should be taken into consideration in cases of patients with free peritoneal fluid (ascites), hyponatraemia, and biochemical patterns of renal failure. In our case, these electrolytic disorders were not obvious and only sodium and calcium levels were slightly decreased. This was likely due to prompt placement of the Foley catheter in the presence of pseudoanuria, the quick deterioration of abdominal pain when the Foley catheter was removed, and normal diuresis observed some hours later when the catheter was replaced. This should have made us suspect bladder injury with peritoneal urinary spilling. As the haematuria and pseudo-anuria were only occasional and temporary, however, we did not suspect a bladder perforation.

\section{Conclusions}

While unrecognised bladder injury during laparoscopy is uncommon, its diagnosis afterwards is often delayed due to the insidious nature of the resultant clinical syndrome and a low suspicion index. Therefore, to prevent bladder injury by an accessory suprapubic trocar, previous vesical voiding and visual control must be maintained without a too-low introduction of the trocar; and if possible, avoiding it. Besides, the entry must be systematically revised from the inside, independently of any previous vesical catheterisation. And for early diagnosis of unrecognised bladder injury, attention must be paid to: a) irritating and persistent abdominal pain of urinary peritonitis, as well as the surgical precedent; b) supposed anuria that is corrected after the positioning of the Foley catheter; c) variable haematuria; d) leukocytosis, eventual electrolytic alterations without fever or other data suggestive of infectious peritonitis, and e) a soft abdomen upon palpation. The key to the diagnosis of bladder injury is awareness of the clinical entity. This diagnosis should be considered in clinically relevant situations, such as those subsequent to laparoscopic surgery.

\section{Authors' Contribution}

JG performed the laparoscopy and analyzed and interpreted the patient data. MA made the diagnosis of bladder perforation and indicated the reintervention. Also analysed and interpreted the patient data. PA helped to laparoscopy, analysed and interpreted the patient data and made the final redaction of the manuscript. All authors read and approved the final manuscript. PA "had full access to all of the data in the study and takes responsibility for the integrity of the data and the accuracy of the data analysis".

\section{Declaration of Interest}

The authors report no declarations of interest.

\section{Funding}

No financial support.

\section{References}

1. Heyns CF, Rimington PD. Intraperitoneal rupture of the bladder causing the biochemical features of renal failure. Br J Urol. 1987;60(3):217-222.

2. Vilos GA, Haebe J, Crumley TL, Maruncic MA, King JH, Denstedt JD. Serum biochemical changes after laparoscopy may be indicators of bladder injury. J Am Assoc Gynecol Laparosc. 2001;8(2):285-290.

3. Kruger PS, Whiteside RS. Pseudo-renal failure following the delayed diagnosis of bladder perforation 
after diagnostic laparoscopy. Anaesth Intensive Care. 2003;31(2):211-213.

4. Tai CK, Li SK, Hou SM, Fan CW. Bladder injury mimicking acute renal failure after cesarean section: a diagnostic challenge and minimally invasive management. Surg Laparosc Endosc Percutan Tech. 2008;18(3):301303.

5. Wang PH, Lee WL, Yuan CC, Chao HT, Liu WM, Yu KJ, Tsai WY, et al. Major complications of operative and diagnostic laparoscopy for gynecologic disease. J Am Assoc Gynecol Laparosc. 2001;8(1):68-73.

6. Mac Cordick C, Lecuru F, Rizk E, Robin F, Boucaya $\mathrm{V}$, Taurelle R. Morbidity in laparoscopic gynecological surgery: results of a prospective single-center study. Surg Endosc. 1999;13(1):57-61.

7. Classi R, Sloan PA. Intraoperative detection of laparoscopic bladder injury. Can J Anaesth. 1995;42(5 Pt 1):415-416.

8. Ostrzenski A, Ostrzenska KM. Bladder injury during laparoscopic surgery. Obstet Gynecol Surv. 1998;53(3):175-180.

9. Jelovsek JE, Chiung C, Chen G, Roberts SL, Paraiso MF, Falcone T. Incidence of lower urinary tract injury at the time of total laparoscopic hysterectomy. JSLS. 2007;11(4):422-427.

10. Lafay Pillet MC, Leonard F, Chopin N, Malaret JM, Borghese B, Foulot H, Fotso A, et al. Incidence and risk factors of bladder injuries during laparoscopic hysterectomy indicated for benign uterine pathologies: a 14.5 years experience in a continuous series of 1501 procedures. Hum Reprod. 2009;24(4):842-849.

11. Azevedo JL, Azevedo OC, Miyahira SA, Miguel GP,
Becker OM, Jr., Hypolito OH, Machado AC, et al. Injuries caused by Veress needle insertion for creation of pneumoperitoneum: a systematic literature review. Surg Endosc. 2009;23(7):1428-1432.

12. Saidi MH, Sadler RK, Vancaillie TG, Akright BD, Farhart SA, White AJ. Diagnosis and management of serious urinary complications after major operative laparoscopy. Obstet Gynecol. 1996;87(2):272-276.

13. Golab A, Slojewski M, Gliniewicz B, Sikorski A. Laparoscopy as a treatment for intraperitoneal bladder injury. Scand J Urol Nephrol. 2003;37(4):339-341.

14. Kyung MS, Choi JS, Lee JH, Jung US, Lee KW. Laparoscopic management of complications in gynecologic laparoscopic surgery: a 5-year experience in a single center. J Minim Invasive Gynecol. 2008;15(6):689-694.

15. Darmon JC, Chevallier L, Diemunsch P, Saussine C, Favreau JJ, Treisser A, Renaud R, et al. [Urinary complications during laparoscopy: a urachal diverticula injury]. Contracept Fertil Sex. 1997;25(5):385-388.

16. Mokoena T, Naidu AG. Diagnostic difficulties in patients with a ruptured bladder. Br J Surg. 1995;82(1):6970.

17. Ratliff DA, Scoble JE. Spontaneous perforation of the bladder presenting as apparent acute renal failure. $\mathrm{Br} \mathrm{J}$ Urol. 1987;60(4):369.

18. Jerwood DC, Mason NP. Pseudo-renal failure after traumatic bladder rupture--the common features. Br J Urol. 1995;76(3):406-407.

19. Wystrychowski A, Nowicki M, Kokot F. Hyponatraemic renal pseudofailure--don't forget the possibility of uroperitoneum. Nephrol Dial Transplant. 1996;11(12):24912492 . 